\title{
Lance-Adams Syndrome
}

\author{
Withanagama CJ, Gamage R, Caldera HPMC \\ National Hospital, Colombo, Sri Lanka
}

Correspondence: Dr.C.J. Withanagama (withanagamachampika@yahoo.com)

\section{Introduction}

Chronic post-hypoxic myoclonus, also known as Lance-Adams syndrome (LAS) is a rare complication of cerebral hypoxia which is seen in survivors of cardiorespiratory arrest following successful CPR. It is characterized by action myoclonus and a cerebellar syndrome which typically occur days to weeks following the hypoxic insult. Worldwide 124 cases have been reported so far after the first case was reported by Lance and Adams in 1963 (1). Here we report a case of LAS.

\section{Case}

A 54-year-old woman who has undergone thyroglossal cyst excision developed stridor followed by cardiorespiratory arrest on post-op day six due to upper respiratory tract obstruction.

CPR was started immediately and succeeded and started on ventilation. She was unconscious for about 24 hours and regained consciousness gradually and was fully conscious and off-ventilation by day 3 .

On regaining of consciousness she was noted to have jerky movements involving the face, upper and lower limbs and trunk. Jerks were precipitated by voluntary movements and stimuli like touch and noise, and disappeared by sleep and relaxation which was diagnosed as action myoclonus. Additionally she developed an incoordination in her upper limbs. She was unable to get up from the bed, sit up from the chair or walk without support due to unsteadiness. Neurological examination revealed gait ataxia, upper limb dysmetria and intentional tremor. Her minimental score was $28 / 30$.

She responded to clonazepam $0.5 \mathrm{mg}$ three times a day and sodium valproate $200 \mathrm{mg}-200 \mathrm{mg}-400 \mathrm{mg}$ with complete resolution of jerks within one week. Gait ataxia and upper limb in coordination improved to near normal over the following 4-6 weeks period.
EEG revealed generalized low amplitude theta activity (Figure 1). T1 and T2 weighted MRI was normal. Six weeks later, cranial Magnetic Resonance Spectroscopy (MRS) showed a moderate decrease in the N-acetyl aspartate (NAA) peaks in bilateral hippocampi (Figure 2) suggesting a neuronal loss due to ischaemia. The NAA / $(\mathrm{Cho}+\mathrm{Cr})$ ratio in the left and right hippocampi were 0.32 and 0.51 respectively (Figure 2). Thus the diagnosis of LAS was established.

\section{Discussion}

Two types of post hypoxic myoclonus can occur in survivors of hypoxic brain injury: the acute and the chronic. The acute form is post-hypoxic myoclonic status epilepticus and the chronic form is LAS.

Lance and Adams in 1963 described four cases of action myoclonus in chronic post-anoxic states. These cases are clinically similar and are characterized by (a) action myoclonus; (b) mild to moderate cerebellar ataxia and (c) very mild intellectual deficit $(2,3)$.

The classic presentation with action myoclonus and cerebellar syndrome in a post-anoxic patient directed the investigators toward the diagnosis of chronic post-hypoxic myoclonus (LAS) which was further supported by MRS findings.

LAS is a distinct entity that may appear after a period of cerebral anoxia, typically following a primary respiratory arrest (3) which occur following regaining consciousness days to weeks after CPR. Myoclonic jerks are typically precipitated by action and stimuli like touch and noise, and it disappears with relaxation of body and during sleep. Cerebellar syndrome involving the trunk and the limbs and mild intellectual impairment are the other manifestations ofLAS. 
Reed et al. (4) demonstrated evidence of medial temporal lobe (hippocampal and parahippocampal) atrophy on MRI brain scans in four patients who suffered acute hypoxic brain injury. Furthermore Zhang et al (1) described a reduced NNA / $(\mathrm{Cho}+\mathrm{Cr})$ ratio in bilateral hippocampi in a patient with LAS. These findings are in keeping with MRS findings of neuronal cell loss in bilateral hippocampi as evidenced by reduced NNA peak and reduced NNA / $(\mathrm{Cho}+\mathrm{Cr})$ ratio in our patient. There are no characteristic EEG abnormalities specific to LAS described in literature. In our patient EEG showed generalized low amplitude theta activity which is compatible with LAS. (Figure 1).

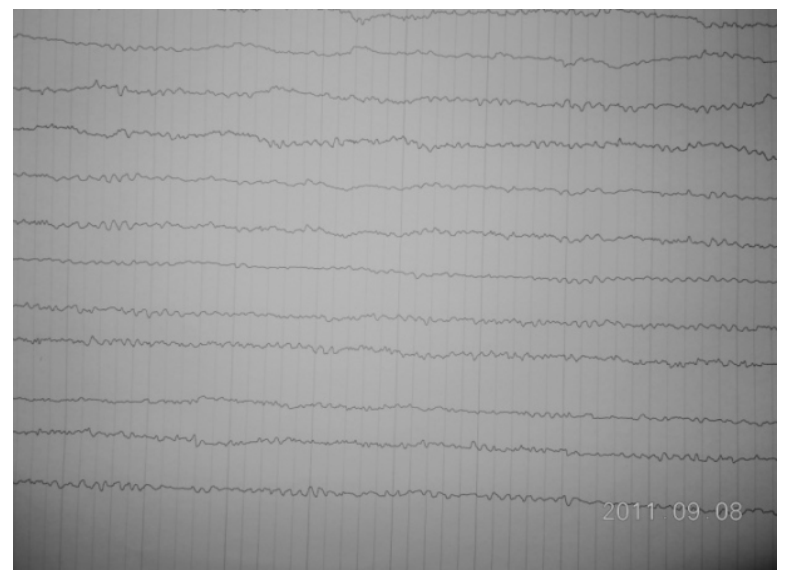

Figure 1: EEG

Although limited data is available LAS shown to have a good prognosis when treated early (5). In a study involving 14 patients with LAS most patients were treated with a combination of clonazepam, valproate and piracetam which resulted in excellent outcome and myoclonus was improved in most of the patients(5). A combination of clonazepam and sodium valproate controlled the myoclonus well in our patient.

The main differential diagnosis for the LAS is post hypoxic myoclonic status epilepticus. It is defined as spontaneous, repetitive, unrelenting, generalised multifocal myoclonus in comatose patients with a recent hypoxic brain insult and has a grave prognosis (6). In contrast, LAS is generally associated with good prognosis and survival with preserved intellect with or without chronic myoclonus and cerebellar features (5).
Authors emphasize the necessity of early diagnosis of this syndrome in order to avoid misinterpretation and early initiation of appropriate treatment. Awareness of its existence is important for the recognition of the syndrome. It is important to distinguish between post-hypoxic myoclonic status epilepticus and LAS, which are two distinct syndromes with very different prognostic implications.

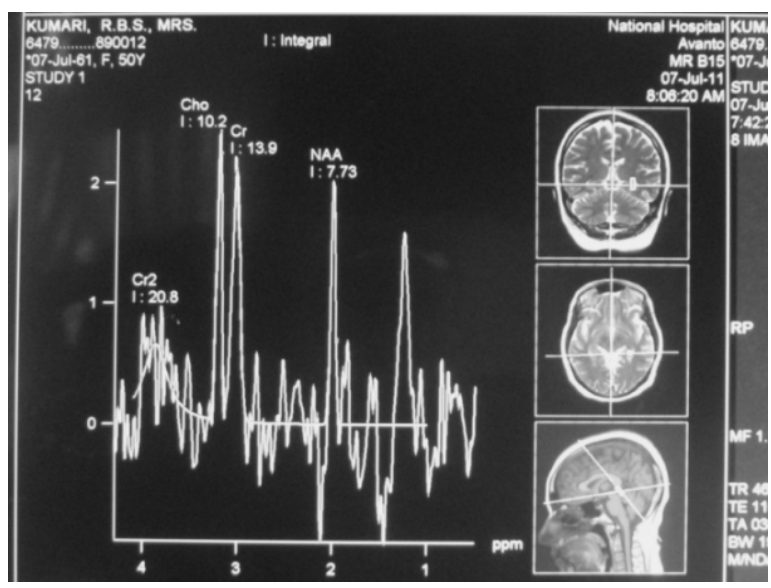

(a)

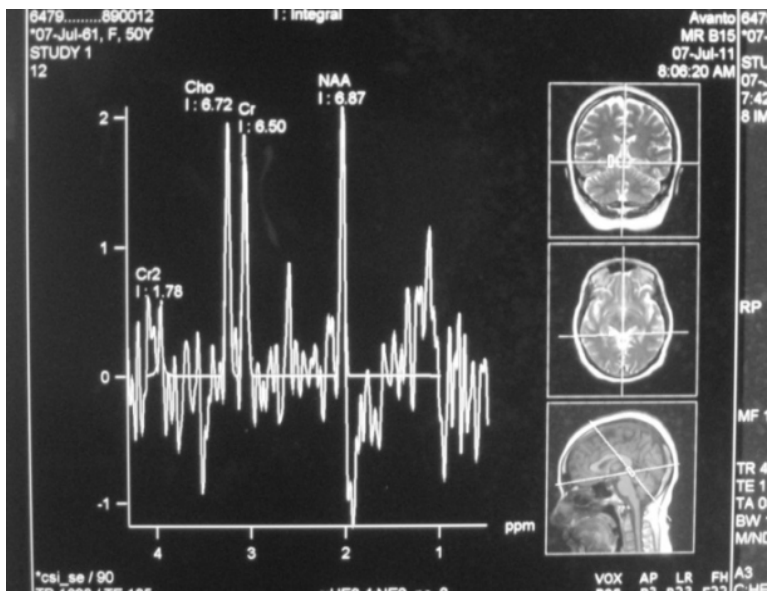

(b)

Figure 2: Six weeks after CPR cranial MRS showed a moderate decrease in NAA peak in the left (a) and right hippocampi (b).

The NAA / $(\mathrm{Cho}+\mathrm{Cr})$ ratio in the left and right hippocampi was 0.32 and 0.51 , respectively 


\section{References}

1. Zhang YX, Liu JR, Jiang B, et al. Lance-Adams Syndrome: a report of two cases. Journal of Zhejiang University Science, 2007; 8: 71520.

2. Russell D. Rollinson, Bernard S. Gilligan. Postanoxic action myoclonus (Lance-Adams syndrome) responding to valproate. Arch Neurol, 1979: 36; 44-45.

3. Kowalczyk EE, Koszewicz MA, Budrewicz SP, Podemski $\mathrm{R}$, Slotwinski K. LanceAdams syndrome in patient with anoxic encephalopathy in the course of bronchial asthma. Wiadomosci Lekarskie, 2006; 59:5602.
4. Reed LJ, Marsden P, Lasserson D. et al. FDG-PET analysis and findings in amnesia resulting from hypoxia. Memory, 1999 Sep-Nov; 7(5-6): 599-612.

5. Werhahn KJ, Brown P, Thompson PD, Marsden CD. The clinical features and prognosis of chronic posthypoxic myoclonus. Mov Disord, 1997 Mar; 12(2): 216-20.

6. Wijdicks EF, Parisi JE, Sharbrough FW. Prognostic value of myoclonus status in comatose survivors of cardiac arrest. Annals of Neurology, 1994; 35: 239-43.

\title{
Posterior Reversible Encephalopathy Syndrome in a patient with pregnancy-induced hypertension in puerperium
}

\author{
Sibly AJM, Caldera HPMC, Gamage R \\ Institute of Neurology, National Hospital of Sri Lanka, Colombo, Sri Lanka
}

Correspondence: Dr.A.J.M. Sibly (mohamedshibly@rocketmail.com)

\section{Introduction}

Posterior reversible encephalopathy syndrome (PRES) is a clinical-neuroradiological entity characterized by headache, vomiting, altered mental status, blurred vision and seizures as well as images suggesting white-gray matter edema involving, in most cases, posterior region of the central nervous system. PRES is commonly associated with hypertensive encephalopathy, preeclampsiaeclampsia, and immunosuppressive and cytotoxic drugs. While usually reversible, the early recognition and treatment of this syndrome is important to prevent permanent neurological sequelae. Treatment is based on the management or withdrawal of the triggering factor. We present a patient who developed pregnancy-induced hypertension after delivery and subsequently PRES, and made an uneventful recovery with prompt management of hypertension and seizures.

\section{Case Report}

Mrs. HS, 30-year-old woman was admitted to Colombo North teaching hospital, with one day history of worsening headache, vomiting, restlessness and altered level of consciousness. There was no history of fever.

She had given birth to her $1^{\text {st }}$ baby seven days earlier. Her antenatal period was uneventful and there was no history of hypertension during that period, at delivery, or after delivery until she was discharged home on the $3^{\text {rd }}$ day after delivery. There was no significant past medical history such as epilepsy, hypertension or connective tissue disorders.

On admission to ETU, she was drowsy and confused with a GCS of 13. There was no neck stiffness. She had mild bilateral pitting ankle edema and her blood pressure was 170/100 $\mathrm{mmHg}$. She had generalized heperreflexia and bilateral upgoing plantars. Examination of her optic fundi was normal. 